\title{
Under-Vaccination in Adults: Qualitative Insights Into Perceived Barriers Reported by Vaccine Supporters, Undecided and Refuters
}

\author{
Zoi Tsimtsiou, et al. [full author details at the end of the article]
}

Accepted: 22 September 2021 / Published online: 17 October 2021

(c) The Author(s), under exclusive licence to Springer Science+Business Media, LLC, part of Springer Nature 2021

\begin{abstract}
Although vaccination is one of the most cost-effective ways of preventing disease, vaccine hesitancy has been included among the ten threats of global health. Addressing low adult vaccination rates requires an adequate understanding of people's views. We explored perceived barriers to immunization among under-vaccinated adults to identify potential differences among vaccine supporters, refuters, and those who are undecided. We conducted a multi-center, mixed-methods study at 23 primary care practices in Greece. Each day, we asked three new randomly-selected adult healthcare users who attended the practice over the course of 30 consecutive working days. We used thematic content analysis to analyze their written answers to open-ended questions that addressed reasons for not getting vaccinated. Out of 1571 participants, two-thirds reported they were under-vaccinated as adults, thus accounting for three out of five of the supporters and the vast majority of the undecided and refuters. "Concerns/fears," a "perception of low susceptibility to disease due to good health status," the "absence of healthcare professional's recommendation," and "previous negative experiences" were four themes common to all three groups. Additional barriers reported by supporters and the undecided included "knowledge gaps about the necessity of adult vaccination," "negligence," and lack of "accessibility." Among refuters, additional themes identified were "mistrust in pharmaceutical companies" and "disbelief in vaccine effectiveness." In conclusion, under-vaccination is common, not only among refuters or the undecided, but also among supporters of adult vaccination. We found similarities and differences in under-vaccinated adults' perceived barriers, depending on their individual perspectives. Physicians and public health services should take into consideration the impact of the wide range of attitudes and beliefs in their effort to address the underlying barriers to vaccination compliance as they attempt to increase vaccination coverage in adults.
\end{abstract}

Keywords Adult immunization · Vaccination hesitancy · Under-vaccination · Mixed-methodology $\cdot$ Attitudes $\cdot$ Barriers 


\section{Introduction}

Although vaccination is one of the most cost-effective ways of preventing disease, vaccine hesitancy presents an international challenge that has been included among the ten threats of global health in 2019 by the World Health Organization (WHO, 2019a). Several factors may influence whether or not a person gets vaccinated (WHO, 2019b). According to the "Increasing Vaccination Model," which was proposed by a WHO global expert group that developed a document entitled "Measuring Behavioral and Social Drivers of Vaccination," what people think and feel, as well as social influences, may affect their motivation to get vaccinated (Brewer et al., 2017). Additional practical factors may affect one's ability to act and get vaccinated, such as vaccine availability, convenience, cost, quality of services, and patient satisfaction, requirements, incentives and intervention fatigue (Brewer et al., 2017; WHO, 2019b). Understanding the reasons for under-vaccination from individuals' own perspectives may help address misinformation, establish confidence in vaccinations, and potentially improve vaccination coverage. While parents' antivaccination attitudes in children have been repeatedly explored, as shown in a recent meta- synthesis study (Díaz Crescitelli et al., 2020), relevant data pertinent to adult vaccinations in general-and not specifically regarding a particular vaccine-are limited (Albright et al., 2017; Eilers et al., 2015; Wheelock et al., 2014).

Previous qualitative studies have investigated individuals' views towards specific vaccines, primarily for seasonal Influenza (Björkman \& Sanner, 2013; Colley, 2008; Cornford \& Morgan, 1999; Evans et al., 2007; Payaprom et al., 2010; Rikin et al., 2018; Sengupta et al., 2004; Siu, 2018; Verger et al., 2018), and also Pneumococcus (Brown et al., 2017; Ridda et al., 2009; Siu, 2018) and Herpes Zoster in older adults (Mortensen, 2011), as well as human papillomavirus in younger women (Rosenthal et al., 2007; Thompson et al., 2018). These studies have identified the main barriers to vaccination as: concerns about its safety (Albright et al., 2017; Brown et al., 2017; Cornford \& Morgan, 1999; Evans et al., 2007; Verger et al., 2018); doubts about its effectiveness (Björkman \& Sanner, 2013; Rikin et al., 2018; Verger et al., 2018; Wheelock et al., 2014); distrust of government and pharmaceutical companies (Eilers et al., 2015); low perception of personal susceptibility (Brown et al., 2017; Payaprom et al., 2010; Siu, 2018; Wheelock et al., 2014); low level of knowledge, especially about specific vaccines for diseases other than influenza (Albright et al., 2017; Wheelock et al., 2014); previous negative experiences (Mortensen, 2011; Verger et al., 2018); and lack of healthcare professionals' recommendations (Evans et al., 2007; Ridda et al., 2009; Rosenthal et al., 2007; Siu, 2018).

It is obvious that individuals' attitudes toward vaccinations are highly variable depending not only on the previously-mentioned factors but also on cultural and infrastructural factors, while significant differences amongst countries have been reported (Dubé et al., 2013). For instance, in a qualitative study in the Netherlands, Poland, and Sweden, although participants overall expressed similar attitudes, important differences were also reported, primarily because of differences in respondents' previous vaccination experiences, the degree of their adherence to social norms, and the degree of their trust in health authorities (Determann et al., 
2016). Considering variations in the capacity of countries to encourage acceptance and their readiness to address hesitancy, WHO encourages all countries to consistently take steps to understand both the extent and the nature of reluctance at a local level (WHO, 2019b). In Greece, even though a cross-sectional telephone survey on influenza vaccine acceptance was administered in 2009 (Sypsa et al., 2009), no published study has provided qualitative data on Greeks' perceived barriers to adult immunization. Moreover, to our knowledge, no previous study internationally has explored potential differences in barriers to overall adult vaccination-relative to a specific vaccine-among people with positive, neutral and negative attitudes towards adults' vaccination.

The aim of this study was to explore the perceived barriers to immunization in adults who reported that they were under-vaccinated and to investigate possible similarities and differences among supporters, those who were undecided, and refuters of adult vaccinations.

\section{Methods}

\section{Study Setting, Design, and Population}

In this multi-center, mixed-methods study, we recruited participants from a population of adults attending 23 primary healthcare practices in Greece (in the regions of Thrace, Macedonia, Thessaly, Central Greece, the Peloponnese, and Crete) for routine care, between March and July 2019. In each practice, we selected three new healthcare users (by using a random number generator), among those who visited the practice each day for 30 consecutive working days, yielding over 2000 recruited healthcare users. Eligibility criteria for the study included those who were at least 18 years, had a sufficient understanding of the Greek language, and signed an informed consent. Exclusion criteria included healthcare users with any severe neurological or mental diseases.

In Greece, all citizens-regardless of their health insurance status-have access to free public healthcare services. They can thus get vaccinated for free for all vaccines that are included in the National Immunization Program. The only prerequisite is a vaccine prescription, which can also be acquired free of charge through public health services. According to the National Immunization Program, certain vaccines are recommended for adults in high-risk groups (e.g., Hepatitis A, Hepatitis B, Varicella Herpes Zoster); the vaccine against Tetanus and Diphtheria is recommended to all adults every ten years, while the Herpes Zoster vaccination is recommended for everyone over 60 years of age. Catch-up doses are recommended to adults depending on their history of past vaccinations or in the absence of evidence of immunization against Measles, Mumps, and Rubella. An influenza vaccine is recommended annually for everyone over 60 years of age and the Pneumococcal disease vaccine is recommended for those aged 65 and older; younger adults who belong to well defined high-risk groups are also included in the recommendations of influenza and pneumococcal vaccines. 


\section{Study Questionnaire, Administration, and Ethical Considerations}

Based on previous literature, we developed a structured questionnaire that included questions in several categories, the first of which included four items specific to participants' socio-demographic characteristics (i.e., age, gender, family status, years of schooling). We then asked a single question on attitudes toward adult vaccination, "What do you believe about vaccination in adults?" which included three response options: "I am in favor of vaccination in adults" (categorized as a supporter), "I am neither in favor nor against adult vaccination" (categorized as an undecided), and "I am against adult vaccination" (categorized as a refuter). In addition, participants reported their medical history (preexisting chronic diseases), and their vaccination history, which included two additional questions: "Did you get a vaccine at least once as an adult?" and "Do you believe that you have completed the recommended vaccinations for your age and health status as an adult?" These two questions could be answered yes, no, or unsure. Those who responded yes to both were considered fully vaccinated participants. Those who responded no or unsure to the latter question were considered under-vaccinated. We included two open-ended questions to explore, among those who believed they had not received all the recommended adult vaccinations, the "under-vaccinated," the reasons why they felt the way they reported: "What's your opinion about the necessity of adult vaccinations?" and "Why didn't you receive all the vaccines that are appropriate for you?"

All primary care physicians consulted the same random number list each day and informed the eligible individuals - according to their order of attendance-about the aims of the study orally. At the end of their visit with their healthcare provider, participants, after providing their written informed consent, completed the written questionnaire, with the exception of those who were illiterate $(n=26)$, for whom researchers conducted the interview and recorded their answers verbatim. Participants placed their completed questionnaires in a box positioned outside the practice in order to ensure anonymity and minimise social desirability bias. The Ethics Committee of the Medical School of Aristotle University of Thessaloniki approved this study (approval reference: 1.38/21-11-18).

We focused our study on under-vaccinated adults. To reiterate, we defined undervaccinated as not having received all the recommended adult vaccinations. We separated our under-vaccinated participants into the following groups: Supporters were those who declared being in favor of vaccination in adults; Undecided participants were those who were neither in favor nor against adult vaccination; and Refuters were those who declared being against adult vaccination.

\section{Data Analyses}

We summarized continuous demographic variables using median and interquartile range (IQR), as well as the range of minimum and maximum values, since they were not normally distributed (Kolmogorov-Smirnov test was performed). We presented categorical variables with absolute numbers and percentages. We used IBM/SPSS 
Statistics Version 21.0 to perform all calculations. Our study focused on under-vaccinated participants who reported they had not received all the recommended adult vaccinations.

We analyzed the open-ended questions using thematic content analysis (Smith, 1992). Our analyses began with open coding describing each section within the data. Using comparisons across the written answers, we refined the open codes into major themes, which provided a coding frame for analysis. We generated ideas and categories after performing line-by-line analyses and we tested and further explored them in subsequent questionnaires until we reached saturation. After compiling a list of meaning statements, we examined emerging clusters of meaningful units. In so doing, we followed the Standards for Reporting Qualitative Research guidelines (O'Brien et al., 2014).

\section{Results}

\section{Participants}

Out of the 2069 eligible healthcare users 1571 agreed to participate (75.9\% response rate). Within the 1571 participants, 1476 participants responded to the question on their attitudes toward adult vaccination, with $1293(87.6 \%)$ reporting that they were supporters of adult vaccination, $76(5.2 \%)$ undecided and 107 (7.2\%) refuters. Among all the supporters, $60.2 \%(n=779 / 1293)$ reported that they were undervaccinated, while the corresponding percentage for the undecided was $85.5 \%$ $(n=65 / 76)$ and for the refuters $91.6 \%(n=98 / 107)$. Within the 942 under-vaccinated participants who reported they had not received all the recommended adult vaccinations (60\% of participants), $82.7 \%$ were supporters, $6.9 \%$ were undecided and $10.4 \%$ were refuters, while 557 had been vaccinated at least once during adulthood: 502 $(64.4 \%)$ of the supporters, $35(53.9 \%)$ of undecided, and $20(20.4 \%)$ of refuters. We present descriptive characteristics of the three under-vaccinated groups by gender (age, years of education, and family status) in Table 1. Further results focus on the under-vaccinated participants.

\section{Perceived Barriers to Adult Vaccination According to the "Increasing Vaccination Model"}

Our study revealed nine themes in total. Taking into consideration the "Increasing Vaccination Model" (Brewer et al., 2017), four of these themes provided insight into what people think and feel (i.e., concerns/fears, perception of low susceptibility to disease, mistrust in pharmaceutical companies, and disbelief in vaccine effectiveness), three related to social processes that affect vaccination decisions (i.e., absence of healthcare professionals' recommendations, knowledge gaps, and previous negative experiences), and two were associated with practical issues (i.e., accessibility barriers and negligence). 
Table 1 Characteristics of supporters of adult vaccination, undecided and refuters, among the under-vaccinated participants by gender

\begin{tabular}{|c|c|c|c|c|c|c|}
\hline \multirow[b]{4}{*}{ Characteristic } & \multicolumn{6}{|c|}{ Under-Vaccinated } \\
\hline & \multicolumn{6}{|l|}{$(n=942)$} \\
\hline & $\begin{array}{l}\text { Supporters } \\
(n=779, \\
82.7 \%)\end{array}$ & & $\begin{array}{l}\text { Undecided } \\
(n=65,6.9 \%)\end{array}$ & & $\begin{array}{l}\text { Refuters } \\
(n=98, \\
10.4 \%)\end{array}$ & \\
\hline & $\begin{array}{l}\text { Males } \\
(n=291)\end{array}$ & $\begin{array}{l}\text { Females } \\
(n=488)\end{array}$ & $\begin{array}{l}\text { Males } \\
(n=27)\end{array}$ & $\begin{array}{l}\text { Females } \\
(n=38)\end{array}$ & $\begin{array}{l}\text { Males } \\
(n=34)\end{array}$ & $\begin{array}{l}\text { Females } \\
(n=64)\end{array}$ \\
\hline \multicolumn{7}{|l|}{ Age } \\
\hline $\begin{array}{l}\text { Median } \\
\text { (IQR; min- } \\
\quad \text { max })\end{array}$ & $\begin{array}{l}65 \\
(20 ; 18-91)\end{array}$ & $\begin{array}{l}61 \\
(20 ; 19-94)\end{array}$ & $\begin{array}{l}63 \\
(23 ; 27-83)\end{array}$ & $\begin{array}{l}68 \\
(16 ; 22-83)\end{array}$ & $\begin{array}{l}68 \\
(19 ; 39-84)\end{array}$ & $\begin{array}{l}65 \\
(16 ; 24-83)\end{array}$ \\
\hline \multicolumn{7}{|c|}{ Years of education } \\
\hline $\begin{array}{l}\text { Median } \\
\qquad \begin{array}{l}\text { (IQR; } \text { min- } \\
\text { max })\end{array}\end{array}$ & $\begin{array}{l}8 \\
(6 ; 0-22)\end{array}$ & $\begin{array}{l}9 \\
(9 ; 0-22)\end{array}$ & $\begin{array}{l}9 \\
(6 ; 0-18)\end{array}$ & $\begin{array}{l}6 \\
(6 ; 0-17)\end{array}$ & $\begin{array}{l}12 \\
(9 ; 0-17)\end{array}$ & $\begin{array}{l}12 \\
(6 ; 0-25)\end{array}$ \\
\hline \multicolumn{7}{|l|}{$\begin{array}{l}\text { Family status } \\
\qquad \begin{array}{l}\text { (\% within } \\
\text { each } \\
\text { group) }\end{array}\end{array}$} \\
\hline Married & $228(29.3)$ & $335(43.0)$ & $20(30.8)$ & $22(33.9)$ & $28(28.6)$ & $38(38.8)$ \\
\hline Single & $40(5.1)$ & $44(5.6)$ & $6(9.2)$ & $5(7.7)$ & $5(5.1)$ & $9(9.2)$ \\
\hline Windowed & $14(1.8)$ & $85(10.9)$ & $1(1.5)$ & $11(16.9)$ & $0(0)$ & $12(12.2)$ \\
\hline Divorced & $9(1.2)$ & $24(3.1)$ & $0(0)$ & $0(0)$ & $1(1.0)$ & $5(5.1)$ \\
\hline
\end{tabular}

In order to reveal possible similarities and differences in perceived barriers to adult vaccinations among supporters, those who were undecided, and refuters, we present in detail the nine themes we found most salient in the three groups of undervaccinated adults.

\section{Common Barriers Among All Groups}

We found four common themes across the supporters' group, the undecided group, and the refuters group: concerns/fears, perception of low susceptibility to disease due to good health status, absence of healthcare professional's recommendation, and previous negative experiences. A narrative of each theme is provided next and supporting quotations from participants are presented in Table 2.

\section{Concerns/Fears}

Our study revealed numerous concerns about the possible consequences of vaccination in terms of immediate side effects or long-term adverse outcomes, including 
Table 2 The four common themes among supporters, undecided and refuters of adult vaccination depicting why they remain under-vaccinated, along with supporting quotations illustrating each theme

Themes Supporting quotations

Concerns/fears

Perception of low susceptibility to disease due to good health status

Absence of health care professionals' recommendations

Previous negative experiences
"I am afraid of the possible side effects." (Undecided, Male, early 70s)

"The long-term side effects of vaccines are unknown." (Refuter, Female, mid 50s)

"I am afraid of possible allergies that may follow a vaccine." (Supporter, Female, early 80 s)

"I suffer from chronic health problems and I am not convinced that they are not going to worsen after vaccination." (Supporter, Female, early 50s)

"I am afraid of whatever I don't know, including new vaccines. I was informed about the flu and the pneumococcal vaccines and I have already done them!" (Undecided, Female, mid 50s)

"I am against vaccination, because vaccines burden our system." (Refuter, Male, mid 50s)

"Getting vaccinated, we insert viruses in our system and we get ill." (Refuter, Female, late 60s)

"I have been informed by the internet that vaccines can cause many diseases like autism, cancer etc." (Refuter, Female, mid 40s)

"I avoid getting vaccinated. I am afraid of needles." (Supporter, Male, early 70s)

"I don't get vaccinated because I feel healthy." (Supporter, Male, late $70 \mathrm{~s})$

"I believe that I am healthy and I don't need them." (Undecided, Male, late 40s)

"I am healthy, apart from some health problems that all the people of my age have, so why should I get vaccinated?" (Refuter, Female, mid 60s)

"I support vaccines in children and I have done it all. As an adult I am against immunization, because I had never got sick. I have a strong immunity system and I truly believe that I will not get ill!” (Refuter, Male, early 70s)

"I haven't done any vaccines because my doctor hasn't advised me to do so." (Supporter, Female, early 60s)

"I decided to not get vaccinated against Herpes Zoster because I was discouraged at the pharmacy." (Supporter, Male, mid 70s)

"No doctor has ever told me to get vaccinated in my adult life." (Undecided, Female, early 40s)

"I am against adult vaccination because of information that I have got from experts." (Refuter, Male, late 60s)

"I was visiting a doctor, one that I trust, and he advised me to better avoid them." (Refuter, Female, late 70s)

"I have been vaccinated once in my life, five years ago. My right arm and chest got swollen. I went to a breast specialist and he told me to go the pharmacy and tell them to cancel the whole batch. I never had another vaccine since then." (Supporter, Female, mid 70s) 
Table 2 (continued)

\begin{tabular}{l} 
Themes \\
\hline Supporting quotations \\
"Whenever I got vaccinated I had very high fever." (Undecided, \\
Female, late 70s) \\
"I have done a vaccine once and it did harm me. I was feeling nausea \\
afterwards. So, I have never done a vaccine again." (Refuter, \\
Female, late 80s)
\end{tabular}

allergies and potential worsening of chronic health problems following a vaccination. The fear of certain vaccines that are not well-known to the general public was also a reason for not pursuing them. Vaccine refuters expressed concerns regarding the safety of vaccines, influenced by anti-vaccination arguments appearing in the media, especially on the internet. Finally, the fear of the needle was also apparent in some adults.

\section{Perception of Low Susceptibility to Disease Due to Good Health Status}

Respondents' perception that they were not susceptible to diseases because they were feeling healthy was very frequently stated as the main reason for declining vaccinations.

\section{Absence of Healthcare Professionals' Recommendations to Get Vaccinated}

Since healthcare professionals, and especially physicians, are the most trusted source of information, the absence of their recommendation to get vaccinated was among the often-stated barriers, especially among the supporters of and those who were undecided about adult vaccinations. Discouragement to get vaccinated with a newer vaccine from healthcare professionals was also reported. Furthermore, the comments from vaccine refuters revealed their direct influence by the negative attitudes of healthcare professionals toward adult vaccination.

\section{Previous Negative Experiences}

Negative prior experiences with vaccinations-mainly regarding mild-to-moderate side effects-were reported, which explained why some supporters, those considered undecided, and refuters declined them. 
Table 3 The three additional themes that have been identified in supporters of adult vaccination and undecided, along with supportive quotations illustrating each theme

\begin{tabular}{ll}
\hline Themes & Supporting quotations \\
\hline $\begin{array}{l}\text { Knowledge gaps about the } \\
\text { necessity of adult vaccina- } \\
\text { tion }\end{array}$ & $\begin{array}{c}\text { "I don't know if I need vaccination and which vaccines I should do." } \\
\text { (Supporter, Female, late 60s) }\end{array}$ \\
& "I didn't know that grownups should get vaccinated!" (Undecided, \\
& Female, early 60s) \\
"I believe that I am still young and I don't need vaccines yet." (Sup- & porter, Female, early 60s) \\
"I don't need them because I am protected by my old age." (Supporter, \\
Male, late 60s) \\
"I am postponing taking this decision, although I know the benefits." \\
(Supporter, Female, early 60s) \\
"I am negligent, that's why." (Undecided, Male early 53s) \\
"I am informed by my doctor, but I am not vaccinated yet. My chil- \\
dren have to take me to the practice when they are not working." \\
(Supporter, Female, late 70s)
\end{tabular}

\section{Other Barriers Identified in Supporters and the Undecided}

Our study identified three additional themes in supporters and undecided: knowledge gaps about the necessity of adult vaccination, negligence, and accessibility barriers. We present supportive quotations illustrating each theme in Table 3.

\section{Knowledge Gaps About the Necessity of Adult Vaccination}

Many supporters and undecided were unaware of the need to be vaccinated as adults, and of the reasons why they should get vaccinated. Moreover, misconceptions about the appropriate ages to get vaccinated for specific vaccines were also revealed.

\section{Negligence}

Supporters and the undecided stated that another reason for postponing vaccination was simply negligence in regard to their health. They had not prioritized their own health care.

\section{Accessibility Barriers}

Only one supporter reported transportation difficulties in reaching a practice.

\section{Other Barriers Identified in Refuters}

We found two additional themes in the refuters: mistrust in pharmaceutical companies and disbelief in vaccine effectiveness. We present supportive quotations in Table 4 . 
Table 4 The two additional themes that have been identified in refuters of adult vaccination, along with supporting quotations illustrating each theme

\begin{tabular}{|c|c|}
\hline Themes & Supporting quotations \\
\hline \multirow{4}{*}{$\begin{array}{l}\text { Mistrust in } \\
\text { pharma- } \\
\text { ceutical } \\
\text { companies }\end{array}$} & "I am mistrustful of pharmaceutical companies." (Refuter, Female, early 50s) \\
\hline & $\begin{array}{l}\text { "I am against vaccines. They don't protect us; they exist just for the profit of pharma- } \\
\text { ceutical companies." (Refuter, Male, mid 50s) }\end{array}$ \\
\hline & $\begin{array}{l}\text { "I am against vaccination because I had read that during vaccination, they put in our } \\
\text { bodies simultaneously- and without our knowledge- other viruses and for some reason } \\
\text { this happens for the profit of pharmaceutical companies." (Refuter, Female, mid 40s) }\end{array}$ \\
\hline & $\begin{array}{l}\text { "I don't know why, but I am against vaccination in adults! I support vaccination in } \\
\text { children, but not in adults, because of what I hear. I don't trust the pharmaceutical } \\
\text { industries." (Refuter, Male, late 60s) }\end{array}$ \\
\hline \multirow{4}{*}{$\begin{array}{l}\text { Disbelief in } \\
\text { vaccine } \\
\text { effective- } \\
\text { ness }\end{array}$} & "With vaccination the odd of getting ill is not zero." (Refuter, Female, early 30s) \\
\hline & $\begin{array}{l}\text { "I am against vaccines because I have read in the internet that there are research data } \\
\text { that prove their ineffectiveness." (Refuter, Female, late } 20 \mathrm{~s} \text { ) }\end{array}$ \\
\hline & $\begin{array}{l}\text { "Although I've done the flu shot this year, I've got sick again." (Refuter, Female, late } \\
60 \mathrm{~s} \text { ) }\end{array}$ \\
\hline & $\begin{array}{l}\text { "I haven't ever vaccinated my children. I used tsikoudia (Greek alcohol drink) on the } \\
\text { head, on the chest. I don't believe in vaccines' value. They fool people." (Refuter, } \\
\text { Female, early 80s) }\end{array}$ \\
\hline
\end{tabular}

\section{Mistrust in Pharmaceutical Companies}

Lack of trust in pharmaceutical companies, reinforced by the belief that financial profit is behind the recommendation of vaccinations, was salient in the majority of refuters. Moreover, unsupported beliefs associated with the financial profit of pharmaceutical companies were also expressed. While adult vaccination was seen as an artificial need, driven by the pharmaceutical industry, some refuters considered vaccination in childhood necessary.

\section{Disbelief in Vaccine Effectiveness}

Vaccine refuters expressed as an argument the fact that vaccines' effectiveness in disease prevention is not one hundred percent guaranteed. Negative views on vaccination appearing in the media, especially on the internet, and often presented as results of valid research, were found to influence the trust in vaccine effectiveness. Moreover, having a personal experience of getting ill, although vaccinated, or knowing people with a similar experience also led to questioning vaccines' effectiveness. A common misconception was the fact that some 
people who had been vaccinated against flu expected that it would protect them against any respiratory infectious disease. Finally, personal beliefs having to do with cultural approaches-like the use of alcohol drinks to massage the skinwere reported by a few refuters to be more effective than vaccines in preventing diseases.

\section{Discussion}

\section{Main Findings}

Our findings provide a better understanding of perceived barriers to adult vaccination through qualitative insight into what people think and feel, and the social processes and practical issues that may diminish adults' motivation to get vaccinated or even deter them altogether from getting vaccinated, according to the "Increasing Vaccination Model" (Brewer et al., 2017; WHO, 2019b). Concerns/ fears, a perception of low susceptibility to disease, the absence of a healthcare professional's recommendations, and previous negative experiences were the four most common themes in all groups. Additional barriers stated by supporters of vaccinations and the undecided included knowledge gaps, negligence, and accessibility barriers. On the other hand, among vaccine refuters, the additional themes that were strongly stated included mistrust in pharmaceutical companies and disbelief in vaccine effectiveness.

Concerns about short- or long-term side effects and potential deterioration of pre-existing chronic diseases, as well as fear of the needle, were stated among vaccine supporters and the undecided. Even stronger concerns about safety, in conjunction with misbeliefs about the serious perceived risks of vaccines, were also found to contribute to vaccine refusal. Our findings are in line with the results of previous qualitative studies and also of a previously conducted telephone survey in Greece, highlighting concerns about safety to be amongst the most frequently stated barriers to adult vaccination internationally (Albright et al., 2017; Björkman \& Sanner, 2013; Brown et al., 2017; Evans et al., 2007; Ridda et al., 2009; Siu, 2018; Sypsa et al., 2009; Teo et al., 2019; Verger et al., 2018).

The perception of low susceptibility to disease due to subjective good health status was also among the most frequent reasons for not getting vaccinated in all groups, along with previous negative experiences. Low perceived risk is also a reason often stated by under-vaccinated individuals (Brown et al., 2017; Nagata et al., 2013; Payaprom et al., 2010; Siu, 2018; Teo et al., 2019; Wheelock et al., 2014). It is apparent that in many under-vaccinated individuals, low perceived risk is associated with knowledge gaps about who should get vaccinated, why and when, apart from personal health beliefs. Similarly, past negative experiences with immunization are also among the documented reasons for avoiding a new vaccination (Mortensen, 2011; Nagata et al., 2013; Teo et al., 2019; Verger et al., 2018). People can easily be stuck in past experiences if they are unwilling to clarify, with the help of healthcare professionals, whether there was an actual problem directly related to the vaccine received and whether that problem could recur. 
The role of healthcare professionals, especially in primary care, seems to be extremely important in influencing their patients' willingness to be vaccinated (WHO, 2015). Our results are congruent with previous qualitative research (Evans et al., 2007; Nagata et al., 2013; Ridda et al., 2009; Rosenthal et al., 2007; Siu, 2018) that suggests that the interaction between healthcare providers and patients is crucial in addressing vaccine hesitancy and refusal (Dubé et al., 2013). Although healthcare professionals are generally strong supporters of vaccination, some of them may hold misperceptions that are similar to those of the public (Nowak et al., 2015). Our findings suggest the need to reinforce the role of primary healthcare professionals in raising awareness in the community and educating their patients regarding vaccinations, especially regarding newer vaccines that may be less known and trusted by the public, like the vaccine against Herpes Zoster or COVID-19. General practitioners are in the unique position to use motivational interviewing and cognitive behavioral therapy to enhance vaccination compliance.

Distrust of pharmaceutical companies was an argument stated by vaccine refuters that has been recently reported in a French qualitative study on flu vaccination in diabetics (Albright et al., 2017). According to the Summary of WHO SAGE on Vaccine Hesitancy, the belief that the pharmaceutical industry is driven by financial motives has created vaccination hesitancy (Nagata et al., 2013). This can extend to distrust in government, if it is perceived that health policy is also being driven by pharmaceutical companies and not by public health interests (Teo et al., 2019). Various pharmaceutical scandals presented in the news media may have reinforced this negative attitude.

Finally, doubts about vaccine effectiveness were expressed as a major barrier by vaccine refuters (Björkman \& Sanner, 2013; Verger et al., 2018; Wheelock et al., 2014). The fact that vaccines are not one hundred percent effective in disease prevention, in combination with the spread of anti-vaccine information in the media and on the internet, plays an important role in influencing the beliefs of refuters. Unfortunately, in social media, anti-vaccine organisations spread the message easily with no oversight or control from health authorities. Moreover, common misconceptions that reinforce distrust in vaccines are related to unrealistic expectations. Addressing knowledge gaps about what to expect when getting vaccinated could reduce disbeliefs in vaccines' effectiveness. Although the association between level of knowledge about vaccination and vaccine acceptance is not straightforward (Dubé et al., 2013), there is evidence that tailored vaccine education can positively impact vaccine knowledge and intentions among adults (Dudley et al., 2021). Interestingly, a recent systematic review and meta-analysis has shown that the shared decision-making process (characterised by patient activation, the bi-directional exchange of information between patients and their healthcare providers and a discussion of available options) can be an effective strategy to increase influenza vaccination rates, especially when it is facilitated by multidisciplinary teams of healthcare professionals (Sanftenberg et al., 2020). 


\section{Study Strengths and Limitations}

To our knowledge, our study is the first to explore differences and similarities in the perceived barriers to immunization among under-vaccinated adults with positive, neutral, and negative attitudes towards vaccination. Moreover, we addressed attitudes towards overall adult immunizations, and not specifically a particular vaccine. Finally, our study is the first to provide qualitative data about people's attitudes towards adult vaccination in Greece, providing evidence that the cultural characteristics of Greeks do not raise barriers to vaccination significantly different from those already reported in the international literature (Dubé et al., 2013).

The main limitation of our study is that we gathered qualitative data as written answers to open-ended questions and not by means of in-depth interviews or focus groups. Using written material represents a methodological limitation because it does not allow for follow-up questions which may lead to a more indepth study of a smaller group. However, we believe that including data from a large and randomly selected population of 1571 adults from 23 practices in 10 different areas outweighed this methodological constraint, since our results confirmed that the vast majority of themes we reported have previously been presented in smaller qualitative studies internationally. Finally, the interpretation of our findings should take into consideration that the barriers we identified have been stated by primary healthcare users and not by the general population, in which barriers related to accessibility may more often be reported.

\section{Conclusions}

Our findings provide evidence that under-vaccination is prevalent not only in refuters or the undecided, but also in supporters of adult immunizations. While there are many similar perceived barriers among under-vaccinated adults in all groups, there are also some important differences between refuters and the other two groups that had not been previously reported: for example, mistrust in pharmaceutical companies and disbeliefs in vaccine effectiveness. Due to the wide range of reasons that affect vaccination rates, multi-component interventions are required to increase vaccination coverage. However, the role of healthcare providers is underlined as especially important in raising awareness in their patients about the importance of adult vaccinations through a patient-centered approach, tailored to each patient's informational needs or concerns. Healthcare providers should regularly recommend that their patients get appropriate vaccines, and explore any previous negative experiences or perception of low susceptibility to disease. Since healthcare professionals can act as role models for their patients, getting vaccinated themselves could help significantly towards building trust even in newly introduced vaccines, like the ones available against the COVID-19 disease. Our results suggest that the wide range of attitudes towards adult vaccination that we have noted should be taken into consideration in any effort to address the underlying barriers of vaccination compliance. 
Acknowledgements The authors gratefully acknowledge Dr Candida Delgatty for editing the manuscript and all the general practitioners that contributed in collecting the data for this study. In particular, we would like to thank: Emmanuil Chatzimanolis, Nikolaos Leotsakos, Konstantinia Karakoula, Alexandra Tsipou, Georgios Zervas, Dimitrios Gougourelas, Dimitrios Kagelidis, Anna Mpotskariova, Androniki Kontou, Ioannis Platakis, Maria Chorti, Xenia Drakou, Georgios Ntrachas, Panagiota Bouga, Thetis Kerasidou, Evaggelia Hartamba, Aristofanis Paganas, Micheal Kalatharas' Panagoula Papadopoulou and Valentina Dislian for their enthusiasm and hard work.

\section{Compliance With Ethical Standards}

Conflict of Interest The authors declare that they have no conflicts of interest.

Ethical Approval The study was approved by the Ethics committee of the Medical School of Aristotle University of Thessaloniki, Greece (Approval Reference: 1.38/21-11-18). All procedures performed in studies involving human participants were in accordance with the ethical standards of the institutional and/or national research committee and with the 1964 Helsinki declaration and its later amendments or comparable ethical standards.

Informed Consent Informed consent was obtained from all individual participants included in the study.

\section{References}

Albright, K., Hurley, L. P., Lockhart, S., Gurfinkel, D., Beaty, B., Dickinson, L. M., et al. (2017). Attitudes about adult vaccines and reminder/recall in a safety net population. Vaccine, 35(52), 7292-7296. https:// doi.org/10.1016/j.vaccine.2017.11.001

Björkman, I., \& Sanner, M. A. (2013). The Swedish A(H1N1) vaccination campaign: Why did not all Swedes take the vaccination? Health Policy, 109(1), 63-70. https://doi.org/10.1016/j.healthpol.2012.09. 004

Brewer, N. T., Chapman, G. B., Rothman, A. J., Leask, J., \& Kempe, A. (2017). Increasing vaccination: Putting psychological science into action. Psychological Science in the Public Interest: A Journal of the American Psychological Society, 18(3), 149-207. https://doi.org/10.1177/1529100618760521

Brown, T., Goldman, S. N., Acosta, F., Garrett, A. M., Lee, J. Y., Persell, S. D., et al. (2017). Understanding Black patients' refusal of pneumococcal vaccination. Journal of Racial and Ethnic Health Disparities, 4(1), 1-8. https://doi.org/10.1007/s40615-015-0193-9

Colley, E. (2008). Influenza vaccination in adults with a long-term condition. Community Practitioner: THe Journal of the Community Practitioners' and Health Visitors' Association, 81(4), 25-28.

Cornford, C. S., \& Morgan, M. (1999). Elderly people's beliefs about influenza vaccination. The British Journal of General Practice: THe Journal of the Royal College of General Practitioners, 49(441), 281-284.

Determann, D., de Bekker-Grob, E. W., French, J., Voeten, H. A., Richardus, J. H., Das, E., et al. (2016). Future pandemics and vaccination: Public opinion and attitudes across three European countries. Vaccine, 34(6), 803-808. https://doi.org/10.1016/j.vaccine.2015.12.035

Díaz Crescitelli, M. E., Ghirotto, L., Sisson, H., Sarli, L., Artioli, G., Bassi, M. C., et al. (2020). A metasynthesis study of the key elements involved in childhood vaccine hesitancy. Public Health, 180, 38-45. https://doi.org/10.1016/j.puhe.2019.10.027

Dubé, E., Laberge, C., Guay, M., Bramadat, P., Roy, R., \& Bettinger, J. (2013). Vaccine hesitancy: An overview. Human Vaccines and Immunotherapeutics, 9(8), 1763-1773. https://doi.org/10.4161/hv.24657

Dudley, M. Z., Taitel, M. S., Smith-Ray, R., Singh, T., Limaye, R. J., \& Salmon, D. A. (2021). Effect of educational and financial incentive-based interventions on immunization attitudes, beliefs, intentions and receipt among close contacts of pregnant women. Vaccine, 39(6), 961-967. https://doi.org/10.1016/j. vaccine.2020.12.067

Eilers, R., Krabbe, P. F., \& de Melker, H. E. (2015). Motives of Dutch persons aged 50 years and older to accept vaccination: A qualitative study. BMC Public Health, 15, 493. https://doi.org/10.1186/ s12889-015-1825-z

Evans, M. R., Prout, H., Tapper-Jones, L. M., \& Butler, C. C. (2007). A qualitative study of lay beliefs about influenza immunisation in older people. The British Journal of General Practice: THe Journal of the Royal College of General Practitioners, 57(538), 352-358. 
Mortensen, G. L. (2011). Perceptions of herpes zoster and attitudes towards zoster vaccination among 50-65-year-old Danes. Danish Medical Bulletin, 58(12), A4345.

Nagata, J. M., Hernández-Ramos, I., Kurup, A. S., Albrecht, D., Vivas-Torrealba, C., \& Franco-Paredes, C. (2013). Social determinants of health and seasonal influenza vaccination in adults $\geq 65$ years: A systematic review of qualitative and quantitative data. BMC Public Health, 13, 388. https://doi.org/10. 1186/1471-2458-13-388

Nowak, G. J., Sheedy, K., Bursey, K., Smith, T. M., \& Basket, M. (2015). Promoting influenza vaccination: Insights from a qualitative meta-analysis of 14 years of influenza-related communications research by U.S. Centers for Disease Control and Prevention (CDC). Vaccine, 33(24), 2741-2756. https://doi.org/ 10.1016/j.vaccine.2015.04.064

O’Brien, B. C., Harris, I. B., Beckman, T. J., Reed, D. A., \& Cook, D. A. (2014). Standards for reporting qualitative research: A synthesis of recommendations. Academic Medicine: Journal of the Association of American Medical Colleges, 89(9), 1245-1251. https://doi.org/10.1097/ACM.0000000000000388

Payaprom, Y., Bennett, P., Burnard, P., Alabaster, E., \& Tantipong, H. (2010). Understandings of influenza and influenza vaccination among high-risk urban dwelling Thai adults: A qualitative study. Journal of Public Health (oxford), 32(1), 26-31. https://doi.org/10.1093/pubmed/fdp086

Ridda, I., Macintyre, C. R., \& Lindley, R. I. (2009). A qualitative study to assess the perceived benefits and barriers to the pneumococcal vaccine in hospitalised older people. Vaccine, 27(28), 3775-3779. https:// doi.org/10.1016/j.vaccine.2009.03.075

Rikin, S., Scott, V., Shea, S., LaRussa, P., \& Stockwell, M. S. (2018). Influenza vaccination beliefs and practices in elderly primary care patients. Journal of Community Health, 43(1), 201-206. https://doi.org/10. 1007/s10900-017-0404-X

Rosenthal, D., Dyson, S., Pitts, M., \& Garland, S. (2007). Challenges to accepting a human papilloma virus (HPV) vaccine: A qualitative study of Australian women. Women and Health, 45(2), 59-73. https://doi. org/10.1300/J013v45n02_04

Sanftenberg, L., Kuehne, F., Anraad, C., Jung-Sievers, C., Dreischulte, T., \& Gensichen, J. (2020). Assessing the impact of shared decision-making processes on influenza vaccination rates in adult patients in outpatient care: A systematic review and meta-analysis. Vaccine, 39(2), 185-196. https://doi.org/10.1016/j. vaccine.2020.12.014

Sengupta, S., Corbie-Smith, G., Thrasher, A., \& Strauss, R. P. (2004). African American elders' perceptions of the influenza vaccine in Durham North Carolina. North Carolina Medical Journal, 65(4), 194-199.

Siu, J. Y. (2018). Perceptions of seasonal influenza and pneumococcal vaccines among older Chinese adults. The Gerontologist. https://doi.org/10.1093/geront/gny139

Smith, C. (1992). Handbook of thematic content analysis. Cambridge University Press.

Sypsa, V., Livanios, T., Psichogiou, M., Malliori, M., Tsiodras, S., Nikolakopoulos, I., et al. (2009). Public perceptions in relation to intention to receive pandemic influenza vaccination in a random population sample: Evidence from a cross-sectional telephone survey. Euro Surveillance: European Communicable Disease Bulletin, 14(49), 19437.

Teo, L. M., Smith, H. E., Lwin, M. O., \& Tang, W. E. (2019). Attitudes and perception of influenza vaccines among older people in Singapore: A qualitative study. Vaccine, 37(44), 6665-6672. https://doi.org/10. 1016/j.vaccine.2019.09.037

Thompson, E. L., Vamos, C. A., Straub, D. M., Sappenfield, W. M., \& Daley, E. M. (2018). Human papillomavirus vaccine information, motivation, and behavioral skills among young adult US women. Journal of Health Psychology, 23(14), 1832-1841. https://doi.org/10.1177/1359105316672924

Verger, P., Bocquier, A., Vergélys, C., Ward, J., \& Peretti-Watel, P. (2018). Flu vaccination among patients with diabetes: Motives, perceptions, trust, and risk culture-a qualitative survey. BMC Public Health, 18(1), 569. https://doi.org/10.1186/s12889-018-5441-6

Wheelock, A., Parand, A., Rigole, B., Thomson, A., Miraldo, M., Vincent, C., \& Sevdalis, N. (2014). Sociopsychological factors driving adult vaccination: A qualitative study. PLOS ONE, 9(12), e113503. https:// doi.org/10.1371/journal.pone.0113503

World Health Organization (WHO) SAGE Vaccine Hesitancy Working Group. (2015). Summary of WHO SAGE conclusions and recommendations on Vaccine Hesitancy. https://www.who.int/immunization/ programmessystems/summary_of_sage_vaccinehesitancy_2pager.pdf?ua=1. Assessed 3 May 2020.

World Health Organization (WHO). (2019b). Improving vaccination demand and addressing hesitancy. https://www.who.int/immunization/programmes_systems/vaccine_hesitancy/en/. Assessed 5 May 2020.

World Health Organization (WHO). (2019a). Ten threats to global health in 2019. https://www.who.int/newsroom/feature-stories/ ten-threats-to-global-health-in-2019. Assessed 5 May 2020. 
Publisher's Note Springer Nature remains neutral with regard to jurisdictional claims in published maps and institutional affiliations.

\section{Authors and Affiliations}

\section{Zoi Tsimtsiou ${ }^{1,2,3}$ (]) Athina Tatsioni ${ }^{2,4} \cdot$ Vasileios Gkizlis ${ }^{2,5}$.}

Konstantinos Kolokas ${ }^{2,6}$. Anastasia Papaioannou ${ }^{2,7}$. Sofia Birka ${ }^{2,3}$. Ilias Tirodimos ${ }^{1} \cdot$ Ioanna Tsiligianni ${ }^{2,8}$

$\triangle$ Zoi Tsimtsiou

zoitsimtsiou@yahoo.gr

1 Department of Hygiene, Social-Preventive Medicine and Medical Statistics, School of Medicine, Aristotle University of Thessaloniki, University Campus, 54124 Thessaloniki, Greece

2 Research Committee of Greek Association of General Practitioners, 54625 Thessaloniki, Greece

3 Health Center of Evosmos, Tegopoulou 2 \& Patron Street, 56224 Thessaloniki, Greece

4 Research Unit for General Medicine and Primary Health Care, Faculty of Medicine, School of Health Sciences, University of Ioannina, 45110 Ioannina, Greece

5 Rural Practice of Mavrommati, 43060 Mavrommati, Karditsa, Greece

6 3Rd Local Health Unit of Evosmos, Kosma Aitolou 1, 56224 Thessaloniki, Greece

7 Health Center of N. Makri, Artemidos \& Marathonos Avenue, 19005 Athens, Greece

8 Department of Social Medicine, Faculty of Medicine, University of Crete, 71003 Heraklion Crete, Greece 